\title{
Asymmetric Amplitude Modulations of Brain Oscillations Generate Slow Evoked Responses
}

\author{
Ali Mazaheri and Ole Jensen \\ F. C. Donders Centre for Cognitive Neuroimaging, Radboud University Nijmegen, NL-6500 HB Nijmegen, The Netherlands
}

\begin{abstract}
Electrophysiological data measured by electroencephalography and magnetoencephalography (MEG) are widely used to investigate human brain activity in various cognitive tasks. This is typically done by characterizing event-related potentials/fields or modulations of oscillatory activity (e.g., event-related synchronization) in response to cognitively relevant stimuli. Here, we provide a link between the two phenomena. An essential component of our theory is that peaks and troughs of oscillatory activity fluctuate asymmetrically; e.g., peaks are more strongly modulated than troughs in response to stimuli. As a consequence, oscillatory brain activity will not "average out" when multiple trials are averaged. Using MEG, we demonstrate that such asymmetric amplitude fluctuations of the oscillatory alpha rhythm explain the generation of slow event-related fields. Furthermore, we provide a physiological explanation for the observed asymmetric amplitude fluctuations. In particular, slow event-related components are modulated by a wide range of cognitive tasks. Hence, our findings provide new insight into the physiological basis of cognitive modulation in event-related brain activity.
\end{abstract}

Key words: electroencephalography; EEG; magnetoencephalography; MEG; event-related potential; ERP; event-related field; ERF; alpha; beta; synchronization

\section{Introduction}

Event-related fields (ERFs) measured using magnetoencephalography (MEG) [analogous to the event-related potentials (ERPs) by using electroencephalography (EEG)] are often applied to investigate neuronal activity associated with the human brain's processing of external events. ERPs/ERFs are calculated by averaging electrophysiological data time-locked to a given stimulus that is repeated multiple times. The ERPs/ERFs contain both fast and slow components. Typically, it is the slow components lasting several hundred milliseconds that are modulated by cognitive tasks, and as such these components are viewed as the link between electrophysiology and cognition. Examples are slow potentials reflecting working memory (Vogel et al., 2005), longterm memory encoding and recognition (Sanquist et al., 1980; Takashima et al., 2006; Rugg and Curran, 2007), action monitoring (Kilner et al., 2004), language comprehension (Kutas and Hillyard, 1980; Hagoort and Brown, 2000), response preparation (Walter et al., 1964), and novelty detection (Soltani and Knight 2000). Although there have been some proposals (Birbaumer et al., 1990; Niedermayer and Lopes Da Silva, 2004; Khader et al., 2008), the exact mechanism for how the slow responses are generated is not well understood.

Electrophysiological signals from the brain have also been investigated by characterizing oscillatory brain activity produced by neuronal synchronization. These modulations are typically

Received April 15, 2008; revised May 24, 2008; accepted June 17, 2008.

This work was supported by the framework of the Netherlands Organization for Scientific Research Innovative Research Incentive Schemes Grant 864.03.007. We thank Rene Scheeringa for constructive comments.

Correspondence should be addressed to Ole Jensen, F.C. Donders Centre for Cognitive Neuroimaging, Radboud University Nijmegen P.0. Box 9101, NL-6500 HB Nijmegen, The Netherlands. E-mail: ole.jensen@fcdonders.ru.nl. D0I:10.1523/JNEUROSCI.1631-08.2008

Copyright $\odot 2008$ Society for Neuroscience $\quad 0270-6474 / 08 / 287781-07 \$ 15.00 / 0$ characterized using event-related synchronization (Pfurtscheller and Lopes da Silva, 1999), temporal spectral evolution (Hari and Salmelin, 1997), or time-frequency representations (TFRs) of power (Tallon-Baudry and Bertrand, 1999). The physiological basis of oscillatory brain activity is better understood than for ERPs/ERFs. Essentially it is the kinetics of the membrane receptors that is thought to determine both synchronization properties and the frequency of the oscillations emerging in a network of coupled neurons (Steriade, 1999; Traub et al., 1999; Jones et al., 2000)

The aim of this MEG study is to demonstrate that amplitude modulations of the ongoing oscillatory activity can explain the generation of slow evoked components. A key element to our hypothesis pertains to how peaks versus troughs of oscillatory activity fluctuate over time. Conventionally, oscillatory activity from the brain is implicitly assumed to have a Gaussian symmetric distribution with respect to peaks and troughs (see Fig. $1 A$ ). In line with the findings of Nikulin et al. (2007), we propose that the amplitude fluctuations of the alpha activity are asymmetric such that the peaks of the alpha oscillations are more strongly modulated than the troughs (or vice versa) (see Fig. $1 B$ ). Several studies have provided evidence that ongoing alpha activity is nonlinear (Stam et al., 1999; Breakspear and Terry 2002). Here, we refine this notion by demonstrating that the distribution of oscillatory activity is skewed because of the asymmetric modulation of peaks and troughs. The asymmetric amplitude modulations have profound consequences when considering event-related averages. Typically, alpha activity is depressed in response to visual stimuli. According to the hypothesis, only the peaks are reduced in magnitude, not the troughs. When the single trials are averaged, the depression in the peaks will result in a negative shift in the calculated ERF (see Fig. 1D). Had the magnitudes of the peaks and 
troughs been symmetrically decreased, no shift in the ERF would have been generated (see Fig. $1 C$ ). In the following, we experimentally establish (1) the existence of asymmetric amplitude modulations in human posterior alpha activity and (2) that these modulations can produce slow ERFs.

\section{Materials and Methods}

In the current study, we developed a simple way to measure the amplitude fluctuation asymmetry (AFA) of a given signal termed the $\mathrm{AFA}_{\text {index }}$. We first used simulated data to assess the properties of the $\mathrm{AFA}_{\text {index }}$. The $\mathrm{AFA}_{\text {index }}$ was applied to MEG data recorded from subjects resting with eyes closed to quantify the amount and direction of the amplitude asymmetry present in spontaneous alpha oscillations. We then applied simple visual stimuli and tested how stimulus-induced modulations in alpha activity correlated with the magnitude of slow ERFs. The direction of the modulation (polarity) was compared with the sign of the $\mathrm{AFA}_{\text {index. }}$

\section{Participants}

Eight normal young adults (three females) with a mean age of 25 (range, 23-28) years participated in the experiment. All participants had normal or corrected-to-normal (better than 6/8) vision.

\section{Recordings}

MEG signals were recorded with a 151 sensor CTF Omega System (VSM MedTech) placed in a magnetically shielded room. In addition, the electrooculogram (EOG) was recorded to later discard trials contaminated by eye movements and blinks. The ongoing MEG and EOG signals were low-pass filtered at $300 \mathrm{~Hz}$, digitized at $1200 \mathrm{~Hz}$, and stored for off-line processing.

\section{Procedure}

Eyes-open/closed task. Subjects were instructed to open and close their eyes according to auditory cues. The auditory cues for the eyes to open and close were, respectively, a single beep $(233 \mathrm{~Hz}, 200 \mathrm{~ms})$ tone and two consecutive beeps ( $500 \mathrm{~ms}$ part). The time between the two types of cues was $7.5 \mathrm{~s}$. To have the strongest possible alpha signal for the analysis, we used only the data epochs in which the eyes were closed.

Visual stimulation. The visual stimuli were contrast gratings (4 cycles/ degree) presented in the lower left visual field with an eccentricity of $3.2^{\circ}$. The width of the circular stimuli extended $5 \times 5^{\circ}$, and the screen was $\sim 70$ $\mathrm{cm}$ away from the subject. The fixation cross was constantly on. Each stimulus was displayed for $0.7 \mathrm{~s}$ and they were presented in four blocks of 150 trials. The intertrial interval varied randomly from 2.5 to $3.5 \mathrm{~s}$. To ensure that participants were attending, they had to respond to a change in the color of the fixation cross by pressing a button with the right index finger. Trials with changes in the fixation cross were ignored in the analysis. Four different contrasts were randomly presented (16, 23, 32, and 64), but only one contrast (32) was used in the analysis.

\section{Data analysis}

Data were analyzed using the Fieldtrip software package (http:// www.ru.nl/fcdonders/fieldtrip/), a Matlab-based toolbox for the analysis of electrophysiological data that has been developed locally. Data were checked for artifacts using a semiautomatic routine that helped detecting and rejecting eye blinks, muscle artifacts, and jumps in the MEG signal caused by the SQUID electronics. Independent component analysis (Bell and Sejnowski, 1995) was used to remove any heart artifacts and eye movements not rejected by the semiautomatic routines (Jung et al., 2000).

The $A F A_{\text {index }}$. To investigate the amplitude asymmetry of the oscillatory activity over time, we developed a measure that quantifies the ratio of the variance of the peaks of an oscillatory activity and the troughs:

$$
\operatorname{AFA}_{\text {index }}=\frac{\operatorname{Var}\left(S_{\text {peaks }}\right)-\operatorname{Var}\left(S_{\text {troughs }}\right)}{\operatorname{Var}\left(S_{\text {peaks }}\right)+\operatorname{Var}\left(S_{\text {troughs }}\right)} .
$$

First, the data were bandpassed at the specific frequency for which the $\mathrm{AFA}_{\text {index }}$ was to be calculated (e.g., $8-12 \mathrm{~Hz}$ ). The time points for the peaks and troughs of the band-passed data were then identified. These time points were used to obtain the signal values of peaks and troughs in

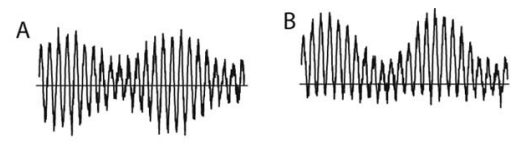

$\overline{100} \mathrm{~ms}$

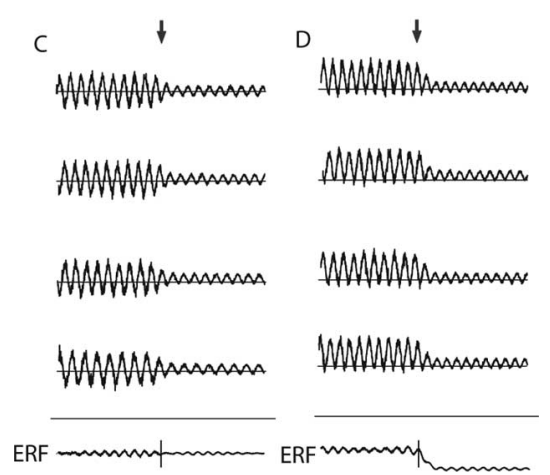

Figure 1. A, The amplitude modulation of neuronal oscillatory activity is conventionally viewed as being symmetric at approximately zero. $\boldsymbol{B}$, We propose that the amplitude modulations of the oscillatory activity are asymmetric such that the peaks are more strongly modulated than the troughs. For the $10 \mathrm{~Hz}$ alpha activity, this could be explained by bouts of activity every $\sim 100 \mathrm{~ms}$. C, The conventional view ignoring asymmetric modulations of oscillatory activity would mean that averaging across trials (the arrow representing the start of the evoked response) would not result in the generation of slow fields. $\boldsymbol{D}$, As a direct consequence of amplitude asymmetry, a depression (or increase) in alpha activity in response to a stimulus will result in the generation of slow fields when multiple trials are averaged.

the raw data. To reduce high-frequency noise, the signal values around peaks and troughs were smoothed using a $10 \mathrm{~ms}$ boxcar kernel (corresponding to a $\sim 100 \mathrm{~Hz}$ low-pass filter). These values were then used to calculate the $\mathrm{AFA}_{\text {index }}$. An $\mathrm{AFA}_{\text {index }}$ close to 0 would mean that the peaks and troughs are modulated similarly, and as such the amplitude fluctuations of the signal are symmetric. A positive $\mathrm{AFA}_{\text {index }}$ would indicate that the peaks are more strongly modulated than the troughs and vice versa. When applying MEG measurements, we predicted that the $\mathrm{AFA}_{\text {index }}$ for fields measured on one side of the current dipole would be positive and negative on the other side (i.e., it would yield a bipolar topography). This bipolar topography is dependent on the orientation of the dipole and specific to the MEG (see the simulation performed in the supplemental material and supplemental Fig. S1, available at www. jneurosci.org). Because the $\mathrm{AFA}_{\text {index }}$ is a quantification of the ratio between peaks and troughs, the zero level (baseline) is of no consequence.

Time-frequency analysis of power. TFRs were obtained using a wavelet transform according to the procedures of Tallon-Baudry et al. (1996). Single trials were convolved by a complex Morlet wavelet: $w\left(t, f_{0}\right)=$ $A \exp \left(-t^{2} / 2 \sigma_{t}^{2}\right) \exp \left(2 i \pi f_{0} t\right)$, where $\sigma_{t}=m / 2 \pi f_{0}$, and $i$ is the imaginary unit. The normalization factor was $A=\left(\sigma_{t} \sqrt{\pi}\right)^{-1 / 2}$. The constant $m$, which defines the compromise between time and frequency resolution, was set to 7 . The wavelet transformation produces a complex time series for the frequencies $f_{0}$ of interest. The TFRs of power were calculated by averaging the squared absolute values of the convolutions over trials. In each subject, we sorted trials according to alpha modulation (power poststimulus minus power prestimulus) based on the data from the MEG sensor with the largest prestimulus alpha amplitude (always a sensor over occipital cortex). The prestimulus and poststimulus intervals, respectively, were -600 to $-100 \mathrm{~ms}$ and $300-800 \mathrm{~ms}$ with respect to stimulus onset.

\section{Results}

\section{Simulations}

To ensure that our measure was not a consequence of a slow DC offset interacting with the alpha rhythm, we investigated various principles of actions using constructed surrogate signals with asymmetric properties as in Figure 1. This first type of interaction 


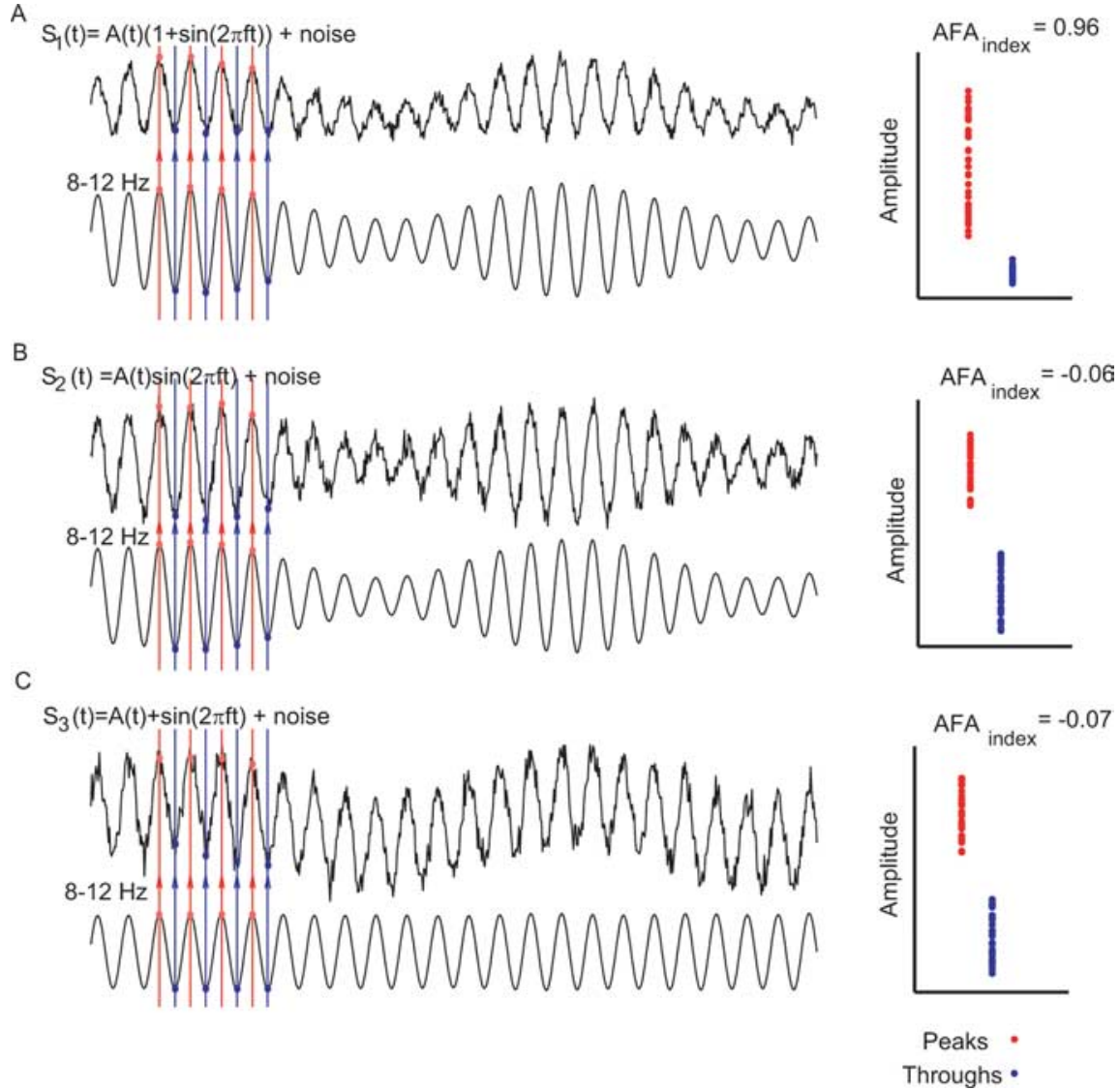

Figure 2. Various simulations in which surrogate signals were used to test the $\mathrm{AFA}_{\text {index }} \cdot \boldsymbol{A}$, The signal $s_{1}(t)$ was designed to have an amplitude asymmetry. The amplitude modulation was determined by a slower signal $A(t)$. Clearly, the peaks (red dots) are more modulated than the troughs (blue dots), yielding a strong $\mathrm{AFA}_{\text {index }} \boldsymbol{B}$, The signal $s_{2}(t)$ was designed such that the slow modulations, $A(t)$, affected the alpha rhythm in a multiplicative manner. Thus, peaks and troughs are modulated symmetrically over time, yielding an $\mathrm{AFA}_{\text {index }}$ close to $0 . \mathrm{C}$, In signal $s_{3}(t)$, slow modulations added to the alpha oscillations (DC-like offset of the signal). This affected peaks and troughs in the same direction, producing an $\mathrm{AFA}_{\text {index }}$ close to 0.

\section{Amplitude asymmetry of ongoing} alpha activity

We performed a whole-head MEG study measuring brain activity during rest and in response to simple visual stimuli. The AFA $_{\text {index }}$ was calculated for 20 epochs of $5 \mathrm{~s}$ for MEG data when subjects were resting with their eyes closed. The topography of alpha power during the eyes-closed task can be seen in Figure 3A. The topography of the $\mathrm{AFA}_{\text {index }}$ in three representative subjects can be seen in Figure $3 B$. All of the subjects demonstrated a bipolar-like topography of the $\mathrm{AFA}_{\text {index }}$, which was particularly pronounced over occipital areas (for topography of all eight subjects, see supplemental Fig. S2 A, available at www. jneurosci.org as supplemental material). This pattern was consistent with what was predicted based on simulations we performed (see the supplemental material, available at www.jneurosci.org). The orientation of the $\mathrm{AFA}_{\text {index }}$ topography (determined by the direction of the magnetic flux, i.e., the magnetic field rotating the current determined by the "right-hand rule") for two subjects suggests a dominant anterior-to-posterior current direction (Fig. 3B, middle, right; as predicted from the simulation in supplemental Fig. S1, available at www.jneurosci.org as supplemental material). This means that peaks were more strongly modulated than troughs to right of the dipole and vice versa to the left. In one other subject, the topography was reversed, suggesting a posteriorto-anterior current direction (Fig. 3B, left). For this subject, it implied that peaks were more strongly modulated than

was designed to produce the hypothesized amplitude asymmetry: $s_{1}(t)=A(t)(1+\sin (2 \pi f t))+$ noise, where $f=10 \mathrm{~Hz}$, and the noise was normally distributed with an SD of 0.2. Note that for illustrative purposes the noise level of the simulated signal is lower than the noise in the measured MEG data. The actual waveform of the slow modulations $A(t)$ is inconsequential but was constructed from a sinusoid with frequency $0.8 \mathrm{~Hz}$ and amplitude 0.5 . The length of the signal was $4 \mathrm{~s}$, and the sampling frequency was $300 \mathrm{~Hz}$. Figure $2 \mathrm{~A}$ shows the resulting signal in which the peaks (red dots) are more strongly modulated than the troughs (blue dots). Applying the $\mathrm{AFA}_{\text {index }}$ to this signal yields 0.96 , i.e., strongly supporting the amplitude asymmetry. We then constructed a signal in which the slow modulations affected the alpha rhythm in a multiplicative manner (Fig. 2B): $s_{2}(t)=$ $A(t) \sin (2 \pi f t)+$ noise. As a consequence, the amplitude of the alpha oscillations is modulated symmetrically over time yielding an $\mathrm{AFA}_{\text {index }}$ close to 0 . Finally, we constructed a signal in which the slow modulation affected the DC offset of the signal in an additive manner (Fig. $2 C): s_{3}(t)=A(t)+\sin (2 \pi f t)+$ noise. This resulted in slow modulations of peaks and troughs in the same direction, producing an $\mathrm{AFA}_{\text {index }}$ close to 0 . These simulations demonstrate that the $\mathrm{AFA}_{\text {index }}$ can successfully detect true asymmetric amplitude fluctuation while being immune to slow multiplicative or additive modulations. troughs to the left of the dipole and vice versa to the right. The emergence of the topography in the asymmetry measure is also illustrated schematically in Figure 6. Averaging the absolute $\mathrm{AFA}_{\text {index }}$ over the subjects revealed the strongest magnitude of the $\mathrm{AFA}_{\text {index }}$ over posterior brain areas in which alpha power was strongest (Fig. $3 C$ ). To ensure that the amplitude asymmetry was specific to the alpha band, we calculated the $\mathrm{AFA}_{\text {index }}$ for frequencies from 5 to $40 \mathrm{~Hz}$ for occipital sensors over all of the subjects (Fig. 3D). The $\mathrm{AFA}_{\text {index }}$ was largest at the alpha frequency range, with a smaller peak associated with more variance in the beta band $(20-24 \mathrm{~Hz})$. We focused our subsequent analysis on the alpha-band activity because it was the dominant rhythm in the posterior areas and had the greatest amplitude asymmetry (for analysis in the beta band, see supplemental Fig. S3, available at www.jneurosci.org as supplemental material).

\section{Relationship between amplitude asymmetry, amplitude modulation, and ERFs}

We then set out to test whether the sign and magnitude of the $\mathrm{AFA}_{\text {index }}$ at rest was consistent with the modulations in the visually evoked ERFs with respect to modulations in alpha power as hypothesized in Figure 1. Using a wavelet approach (TallonBaudry et al., 1996), we calculated TFRs of power during a simple visual stimulation task. The modulation in alpha power with re- 
spect to a prestimulus baseline $\left(P_{\text {modulation }}\right.$ $=P_{\text {post }}-P_{\text {pre }} ; P_{\text {pre }}:-0.6<t_{\text {pre }}<-0.1 \mathrm{~s}$; $P_{\text {post }}: 0.3<t_{\text {post }}<0.8 \mathrm{~s}$ ) after the visual stimulus was calculated for each trial. The trials were then sorted in three bins according to the magnitude of the modulation. Figure $4 A$ shows the TFRs for the bins with the lowest and highest alpha modulations for a representative posterior sensor. We then characterized the modulation in ERF amplitude with respect to alpha modulations. This was done by sorting the trials in three bins according to the magnitude of alpha modulation. The ERFs were then calculated for the trials in each bin. Linear fits of ERF amplitude (0.3-0.8 s) versus the alpha modulation $\left(P_{\text {modulation }}\right)$ for the three bins were then calculated for all sensors. The slope of the fitted lines represented the rate and direction of ERF modulation as a result of alpha-power modulation (for a graphical illustration, see supplemental Fig. S4, available at www.jneurosci.org as supplemental material). The slopes were normalized between -1 and 1 by dividing by the largest absolute slope in each subject. A negative slope means that the ERF amplitude is decreasing with alpha modulation; a positive slope means that the ERF amplitude is increasing with alpha modulation (for the slope topography of all eight subjects, see supplemental Fig. S2 $B$, available at www.jneurosci.org as supplemental material). Note that what is essential here are the signs of the slopes when relating the modulations in alpha power to the ERFs. For a discussion on the signs of the actual deflections of the ERFs in relation to alpha power, see supplemental Figures S5-S7 (available at www.jneurosci.org as supplemental material). Topographic representations of the slopes are shown for the three representative subjects in Figure $4 B$ (same subjects as in Fig. 3). An important clue is that the modulation is reversed for the first subject compared with the last two as was the case for the $\mathrm{AFA}_{\text {index }}$ (Fig. $3 B$ ). The grand average of the absolute inflections in the slow component of the ERF is clearly constrained to the posterior part of the brain (Fig. 4C). These findings demonstrate a strong correlation between the slow components of the ERFs and stimulus-induced modulation of alpha power.

We then asked whether the $\mathrm{AFA}_{\text {index }}$ could account for the correlation between modulation in alpha power and the slow components of the visually evoked responses. To statistically quantify this, we did a binomial sign test between the direction of the $\mathrm{AFA}_{\text {index }}$ and the sign of the ERF inflection with alpha modulation. The topography of consistency (quantified by a sign test) between the $\mathrm{AFA}_{\text {index }}$ calculated during eyes closed and the direction of ERF inflection with alpha modulation is shown in Figure $5 A$. Using the binomial test, we determined the probability of observing matching signs of the ERF modulation and the $\mathrm{AFA}_{\text {index }}$ under the null hypothesis that the signs were unrelated.
A

B

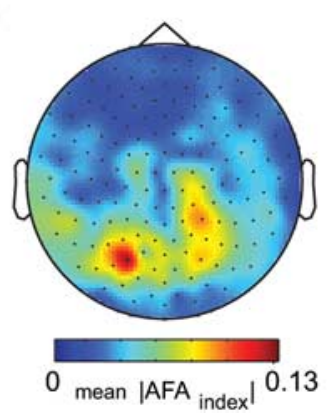

S2
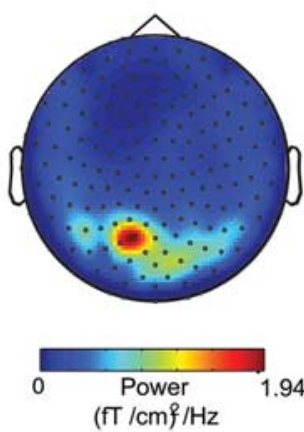

S3
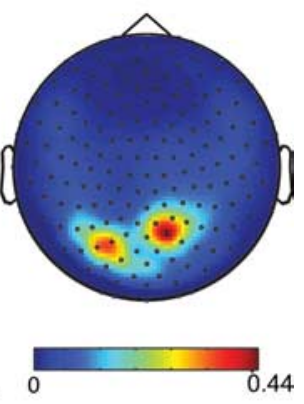

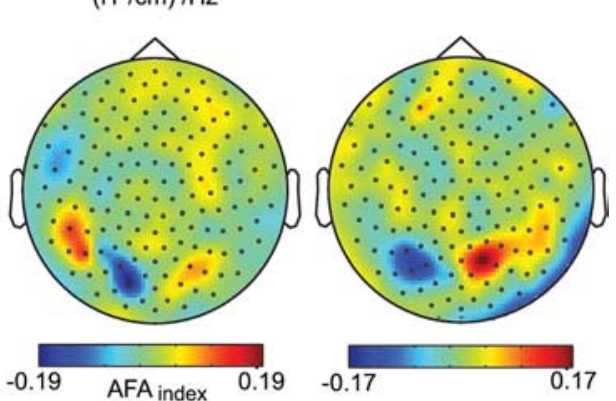

$\mathrm{D}$

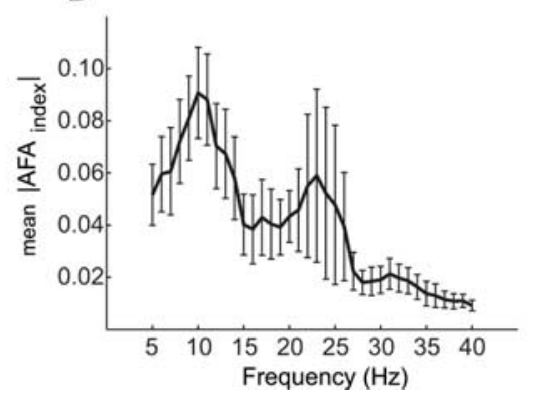

Figure 3. Asymmetry of the alpha amplitude fluctuations. $\boldsymbol{A}$, The topography of alpha power during eyes closed in three representative subjects. $\boldsymbol{B}$, The $A F A_{\text {index }}$ applied to the eyes closed data in three representative subjects. The topography of the $A F A_{\text {index }}$ resembles a bipolar distribution as predicted (see supplemental Fig. S1, available at www.jneurosci.org as supplemental that the largest values over posterior brain areas are consistent with where the alpha power was dominant. $\boldsymbol{D}$, The absolute $A F A_{\text {index }}$ for frequencies from 5 to $40 \mathrm{~Hz}$ for occipital sensors averaged over the subjects. The $A F A_{\text {index }}$ peaked at the alpha $(\sim 10 \mathrm{~Hz})$ and beta $(\sim 23 \mathrm{~Hz})$ frequencies. Error bars denote SEM.

Matching signs in more than six of the eight subjects were considered significant $(p<0.035)$. Note the two clusters of significant sensor locations over posterior areas; the clustering makes it unlikely that the significant effect is caused by multiple comparisons. Finally, when relating the $\mathrm{AFA}_{\text {index }}$ to the magnitude of the ERF modulation over subjects, we found a very strong correlation (seven posterior sensors; $r=0.80 ; p=$ $0.018)$. The data subjected to the same analysis in the beta band $(\sim 23 \mathrm{~Hz})$ did not reveal a significant relationship between the slow ERF components and modulations in the beta power (supplemental text and supplemental Fig. S3, available at www.jneurosci.org as supplemental material). To conclude, we demonstrated that amplitude fluctuations of the alpha activity are asymmetric (as seen in the resting eyes closed data) and, consequently, the stimulus-induced amplitude modulations of such activity generate slow components of the eventrelated fields that are correlated in sign and magnitude to the asymmetric amplitude fluctuations observed during rest. 
A
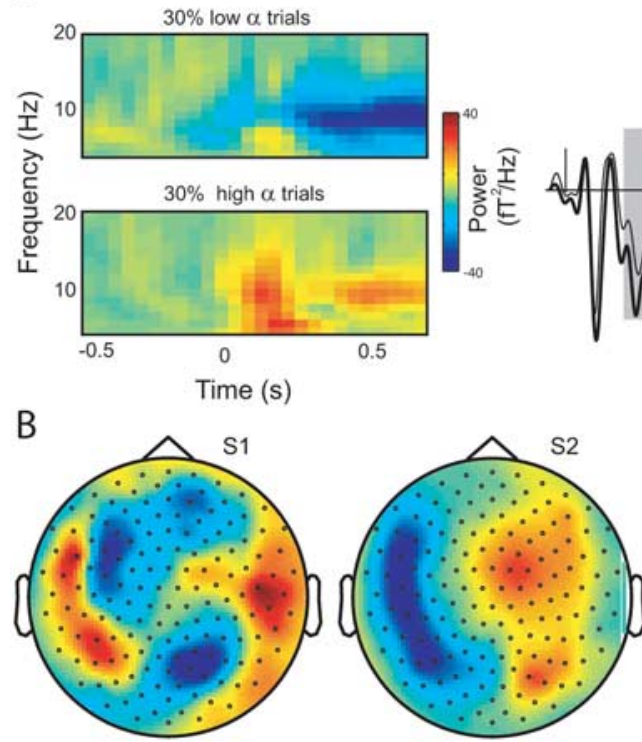

C

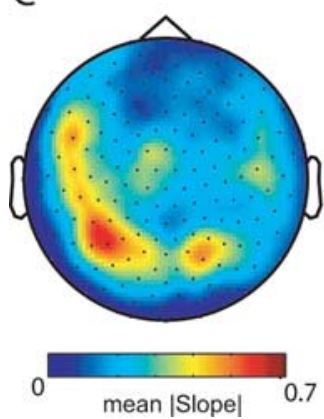

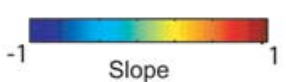

(ERF change with power)
$30 \%$ low $\alpha$ trials $30 \%$ high $\alpha$ trials $50 \mathrm{fT}$

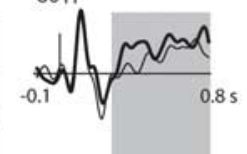

Figure 4. A, TFRs of the trials with the $30 \%$ lowest and $30 \%$ highest modulations of alpha power (TFRs baseline corrected; $-0.6<t<-0.1 \mathrm{~s}$ ) in a representative subject. The respective ERFs (right) reveal a clear difference in the sustained modulation with respect to low- (thin line) and high-alpha-power changes (thick line). $\boldsymbol{B}$, The topography of the ERF modulation with respect to poststimulus alpha power from the TFRs. The color represents the slope resulting from linear fit between ERF $(0.3-0.8 \mathrm{~s})$ and alpha power $(0.3-0.8 \mathrm{~s})$. The bipolar distributions are consistent with supplemental Fig. S1 (available at www.jneurosci.org as supplemental material). C, The grand average of absolute ERF modulations with alpha power averaged across eight subjects.

\section{Discussion}

In this study, we identified a mechanism by which event-related fields can be produced from oscillatory brain activity. We demonstrated that amplitude fluctuations of ongoing oscillations with respect to peaks and troughs are not symmetrically modulated. Because of this asymmetry, slow ERFs are produced from the amplitude modulations of the $\sim 10 \mathrm{~Hz}$ alpha activity in response to visual stimuli. It is important to note that the amplitude fluctuations of the oscillatory activity can remain asymmetric throughout the trial; however, it is primarily the asymmetric modulations in response to a stimulus that are important here. Had the magnitudes of the peaks and troughs been symmetrically decreased, no shift in the ERF would have been generated (Fig. 1C). Our findings support studies by, for example, Stam et al. (1999) and Breakspear and Terry (2002), challenging the conventional view that ongoing EEG/MEG signals are linear with a Gaussian distribution.

Although our analysis is based on MEG data, the basic assumptions hold for EEG data as well. Indeed the physiological mechanisms producing the EEG and MEG signals are highly related. EEG mainly measures scalp potentials produced by neuronal return currents after excitatory synaptic input, whereas MEG mainly measures the primary dendritic currents (Hämäläinen et al., 1993). It should be mentioned that we have chosen MEG to provide the proof of principle because the orientations of the bipolar patterns allow us to establish the connection between alpha amplitude asymmetry and the direc-
A

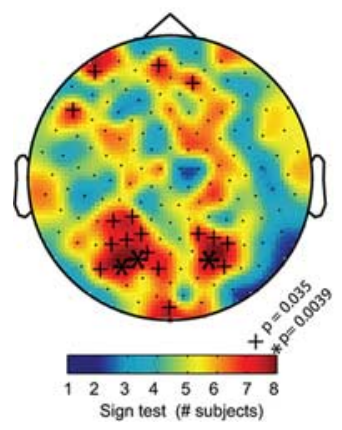

B

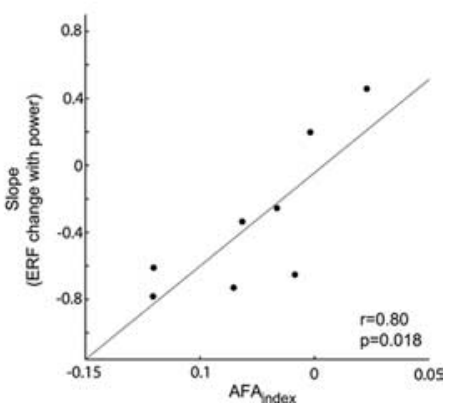

Figure 5. Correlation between alpha amplitude asymmetry and the modulations of slow ERFs with alpha power. $A$, The consistency between the sign of the $\mathrm{AFA}_{\text {index }}$ (from eyes closed data) and the sign of the modulation in slow visually evoked ERF with alpha power. The color code represents the number of consistent signs over the eight subjects. More than six consistent signs are considered significant (binomial test). Note the clustering of adjacent sensors with significant effects over posterior areas. $\boldsymbol{B}$, The correlation between the $\mathrm{AFA}_{\text {index }}$ and the slope of slow ERF modulation with alpha power (the seven posterior sensors with the largest $\mathrm{AFA}_{\text {index }}$ in Fig. 30. The strong correlation strongly suggests that the slow modulations in the ERFs are produced by changes in asymmetric amplitude changes of alpha power. tion of modulations of the ERFs (Fig. 5). Given the spatial smearing of EEG data, it would be difficult to make use of bipolar scalp distributions; however, one can still calculate the $\mathrm{AFA}_{\text {index }}$ and correlate it to the changes of slow ERPs with oscillatory modulation.

The mechanism proposed does in no way discount the value of conventional ERP/ERF analysis. Although our model can explain the slow components of ERFs, the physiological mechanism accounting for the fast components of the ERPs/ERFs requires a different model (Jones et al., 2007). Furthermore, although slow ERP/ERF components can be produced by modulations in oscillatory activity is does not mean that the conventional ERP/ERF analysis is inappropriate as a tool in cognitive neuroscience. The mechanism we propose does however call for some reinterpretation of slow evoked responses. Typically, these responses are seen as additive reflecting a cognitive process "turning on." Our model suggests that slow evoked responses are a consequence of a change in the brain state indexed by oscillatory activity. This makes prestimulus activity just as important as poststimulus activity.

Our current study focused on posterior alpha activity and how it relates to the modulation of the slow components of visual 
ERFs. Posterior alpha activity has been shown to be modulated by cognitive manipulations in a wide variety of tasks. This includes working memory operations (Klimesch et al., 1996; Krause et al., 1996; Jensen et al., 2002; Jokisch and Jensen, 2007; Medendorp et al., 2007), long-term memory retrieval (Klimesch, 1999; Babiloni et al., 2004), directed attention (Worden et al., 2000), and language comprehension (Bastiaansen and Hagoort 2006). In future work, it would be interesting to relate the modulations in alpha activity to the slow components that have been found to be modulated in related tasks (Kutas and Hillyard, 1980; Sanquist et al., 1980; Hagoort and Brown, 2000; Vogel et al., 2005; Takashima et al., 2006; Rugg and Curran, 2007). It is possible that the formation of these ERFs is caused by changes in the alpha-band activity.

We have proposed a simple physiological mechanism that is responsible for generating the amplitude asymmetry. It is accepted that the magnetic fields recorded by MEG are produced mainly by intracellular currents in the dendrites of pyramidal cells (Hämäläinen et al., 1993) (Fig. 6A). A key element of our proposal is that the dominating dendritic currents producing the magnetic fields primarily are inward, i.e., running from the distal synapses to the soma. Although there might be outward dendritic currents as well because of depolarization around the soma, it is unlikely that these currents would have the same magnitude as the inward currents. Thus, the measured oscillatory alpha activity is most easily explained by bouts of inward dendritic currents produced every $\sim 100 \mathrm{~ms}$. Note that the outward dendritic currents should not be confused with the instantaneous return (volume) current. The consequences for the topography of the amplitude asymmetry are illustrated in Figure 6, $C$ and $D$ (see also simulations in the supplemental material and supplemental Fig. S1, available at www.jneurosci.org). We do not mean to imply that the inhibitory inputs are unimportant for generating the alpha rhythm. Indeed, Jones et al. (2000) have proposed a physiologically realistic model for alpha oscillations in which GABAergic inhibition plays a crucial role for the rhythm generation. Likewise thalamic input to granular and intragranular layers has also been shown to play an essential role in generating the neocortical alpha rhythm (Hughes and Crunelli 2005). Although these elements are important for rhythm generation, our arguments on amplitude asymmetry pertain to the inward dendritic currents directly producing the magnetic fields.

Although we focused the analysis on the posterior alpha activity, the proposed mechanism might generalize to other frequency bands and brain regions. For instance Nikulin et al. (2007) demonstrated a correlation between low-frequency drifts and the $\sim 10 \mathrm{~Hz}$ somatosensory $\mu$-rhythm. Consistent with our proposal, they interpreted their findings as a consequence of amplitude asymmetry and proposed that resulting baseline shifts could account for the formation of somatosensory evoked responses. There is no reason for why the amplitude asymmetry would be constrained to alpha-band activity. Indeed, cognitive modulations have been observed in both slower (delta, theta) and faster (beta, gamma) frequency bands (Klimesch, 1999; Bacşar et al., 2000; Kahana et al., 2001; Bastiaansen and Hagoort 2006; Jensen et al., 2007). In particular, gamma-band activity is interesting. Given that the period of a gamma cycle is $10-30 \mathrm{~ms}$, it is possible that asymmetric amplitude modulations can account for some of the faster modulations in ERFs (e.g., the N1, P1, N2, P2). Future work is required to investigate whether the principle of amplitude asymmetry and generation of ERPs/ERFs generalizes to frequency bands beyond the alpha range.
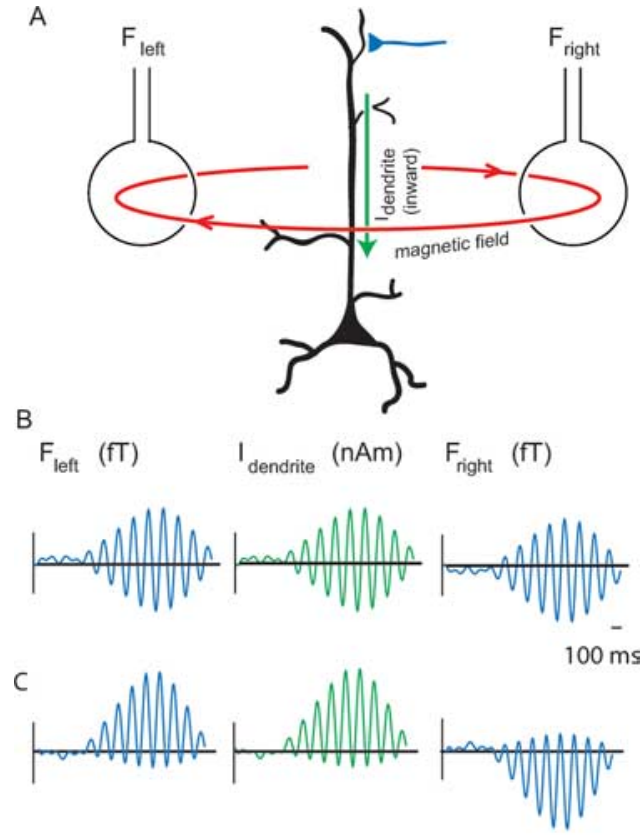

D

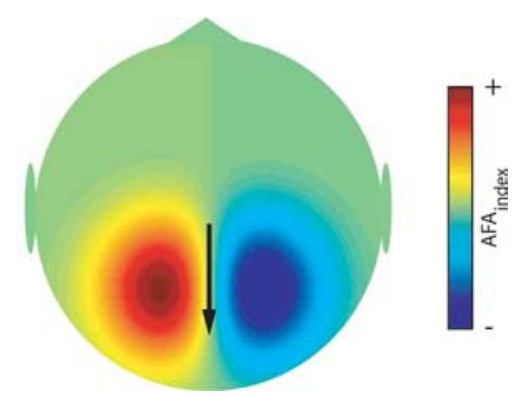

Figure 6. A, MEG signals measured outside the human scalp are primarily thought to be produced by magnetic fields induced by dendritic intracellular currents $\left(l_{\text {dendrite }}\right)$ in pyramidal cells. The polarity of the field will be determined by the direction (inward or outward) of the currents in the dendrites. The two coils represent the sensors outside the head measuring the fields to the left and right of the source $\left(F_{\text {left }} F_{\text {right }}\right)$. B, The amplitude modulation of neuronal oscillatory activity is conventionally viewed as being symmetric at approximately zero. This implies that inward and outward dendritic currents are of the same magnitude. As a consequence, the amplitude modulation measured by the sensors is symmetric as well. C, We propose that the amplitude modulations of the oscillatory activity are asymmetric such that the peaks are more strongly modulated than the troughs. For the $10 \mathrm{~Hz}$ alpha activity, this is explained by bouts of inward dendritic currents every $\sim 100 \mathrm{~ms}$. As a consequence, amplitude modulations are positive (peaks more strongly modulated than troughs) in magnetic fields measured to the left of the source and negative (troughs modulated stronger peaks) in fields measured to the right. $\boldsymbol{D}$, The amplitude modulation as characterized by the $\mathrm{AFA}_{\text {index }}$ would produce a bipolar pattern.

\section{References}

Babiloni C, Babiloni F, Carducci F, Cappa S, Cincotti F, Del Percio C, Miniussi C, Moretti DV, Pasqualetti P, Rossi S, Sosta K, Rossini PM (2004) Human cortical EEG rhythms during long-term episodic memory task. A high-resolution EEG study of the HERA model. Neuroimage 21:1576-1584.

Bacşar E, Başar-Eroğlu C, Karakaş S, Schürmann M (2000) Brain oscillations in perception and memory. Int J Psychophysiol 35(2-3):95-124.

Bastiaansen M, Hagoort P (2006) Oscillatory neuronal dynamics during language comprehension. Prog Brain Res 159:179-196.

Bell AJ, Sejnowski TJ (1995) An information maximisation approach to blind separation and blind deconvolution. Neural Comput 7:1129-1159.

Birbaumer N, Elbert T, Canavan AG, Rockstroh B (1990) Slow potentials of the cerebral cortex and behavior. Physiol Rev 70:1-41.

Breakspear M, Terry JR (2002) Detection and description of non-linear in- 
terdependence in normal multichannel human EEG data. Clin Neurophysiol 113:735-753.

Hagoort P, Brown CM (2000) ERP effects of listening to speech compared to reading: the P600/SPS to syntactic violations in spoken sentences and rapid serial visual presentation. Neuropsychologia 38:1531-1549.

Hämäläinen MS, Hari R, Ilmoniemi RJ, Knuutila J, Lounasmaa (1993) : Magnetoencephalography. Theory, instrumentation and applications to the noninvasive study of brain function. Rev Mod Phys 65:413-497.

Hari R, Salmelin R (1997) Human cortical oscillations: a neuromagnetic view through the skull. Trends Neurosci 20:44-49.

Hughes SW, Crunelli V (2005) Thalamic mechanisms of EEG alpha rhythms and their pathological implications. Neuroscientist 11:357-372.

Jensen O, Gelfand J, Kounios J, Lisman JE (2002) Oscillations in the alpha band $(9-12 \mathrm{~Hz})$ increase with memory load during retention in a shortterm memory task. Cereb Cortex 12:877-882.

Jensen O, Kaiser J, Lachaux JP (2007) Human gamma-frequency oscillations associated with attention and memory. Trends Neurosci 30:317-324.

Jokisch D, Jensen O (2007) Modulation of gamma and alpha activity during a working memory task engaging the dorsal or ventral stream. J Neurosci 27:3244-3251.

Jones SR, Pinto DJ, Kaper TJ, Kopell N (2000) Alpha-frequency rhythms desynchronize over long cortical distances: a modeling study. J Comput Neurosci 9:271-291.

Jones SR, Pritchett DL, Stufflebeam SM, Hämäläinen M, Moore CI (2007) Neural correlates of tactile detection: a combined magnetoencephalography and biophysically based computational modeling study. J Neurosci 27:10751-10764.

Jung TP, Makeig S, Westerfield M, Townsend J, Courchesne E, Sejnowski TJ (2000) Removal of eye activity artifacts from visual event-related potentials in normal and clinical subjects. Clin Neurophysiol 111:1745-1758.

Kahana MJ, Seelig D, Madsen JR (2001) Theta returns. Curr Opin Neurobiol 11:739-744.

Khader P, Schicke T, Röder B, Rösler F (2008) On the relationship between slow cortical potentials and BOLD signal changes in humans. Int J Psychophysiol 67:252-261.

Kilner JM, Vargas C, Duval S, Blakemore SJ, Sirigu A (2004) Motor activation prior to observation of a predicted movement. Nat Neurosci 7:1299-1301.

Klimesch W (1999) EEG alpha and theta oscillations reflect cognitive and memory performance: a review and analysis. Brain Res Brain Res Rev 29:169-195.

Klimesch W, Schimke H, Doppelmayr M, Ripper B, Schwaiger J, Pfurtscheller G (1996) Event-related desynchronization (ERD) and the Dm effect: does alpha desynchronization during encoding predict later recall performance? Int J Psychophysiol 24:47-60.

Krause CM, Lang AH, Laine M, Kuusisto M, Pörn B (1996) Event-related EEG desynchronization and synchronization during an auditory memory task. Electroencephalogr Clin Neurophysiol 98:319-326.
Kutas M, Hillyard SA (1980) Reading senseless sentences: brain potentials reflect semantic incongruity. Science 207:203-205.

Medendorp WP, Kramer GF, Jensen O, Oostenveld R, Schoffelen JM, Fries P (2007) Oscillatory activity in human parietal and occipital cortex shows hemispheric lateralization and memory effects in a delayed double-step saccade task. Cereb Cortex 17:2364-2374.

Niedermayer E, Lopes Da Silva F (2004) Electroencephalography: basic principles, clinical applications and related fields. Baltimore: Williams and Wilkins.

Nikulin VV, Linkenkaer-Hansen K, Nolte G, Lemm S, Müller KR, Ilmoniemi RJ GC (2007) A novel mechanism for evoked responses in the human brain. Eur J Neurosci 25:3146-3154.

Pfurtscheller G, Lopes da Silva FH (1999) Event-related EEG/MEG synchronization and desynchronization: basic principles. Clin Neurophysiol 110:1842-1857.

Rugg MD, Curran T (2007) Event-related potentials and recognition memory. Trends Cogn Sci 11:251-257.

Sanquist TF, Rohrbaugh JW, Syndulko K, Lindsley DB (1980) Electrocortical signs of levels of processing: perceptual analysis and recognition memory. Psychophysiology 17:568-576.

Soltani M, Knight RT (2000) Neural origins of the P300. Crit Rev Neurobiol 14:199-224.

Stam CJ, Pijn JP, Suffczynski P, Lopes da Silva FH (1999) Dynamics of the human alpha rhythm: evidence for non-linearity? Clin Neurophysiol 110:1801-1813.

Steriade M (1999) Coherent oscillations and short-term plasticity in corticothalamic networks. Trends Neurosci 22:337-345.

Takashima A, Jensen O, Oostenveld R, Maris E, van de Coevering M, Fernández G (2006) Successful declarative memory formation is associated with ongoing activity during encoding in a distributed neocortical network related to working memory: a magnetoencephalography study. Neuroscience 139:291-297.

Tallon-Baudry C, Bertrand O (1999) Oscillatory gamma activity in humans and its role in object representation. Trends Cogn Sci 3:151-162.

Tallon-Baudry C, Bertrand O, Delpuech C, Pernier J (1996) Stimulus specificity of phase-locked and non-phase-locked $40 \mathrm{~Hz}$ visual responses in human. J Neurosci 16:4240-4249.

Traub RD, Jefferys JG, Whittington MA (1999) Fast oscillations in cortical circuits. Cambridge, MA: MIT.

Vogel EK, McCollough AW, Machizawa MG (2005) Neural measures reveal individual differences in controlling access to working memory. Nature 438:500-503.

Walter WG, Cooper R, Aldridge VJ, McCallum WC, Winter AL (1964) Contingent Negative Variation: An Electric Sign of Sensorimotor Association and Expectancy in the Human Brain. Nature 203:380-384.

Worden MS, Foxe JJ, Wang N, Simpson GV (2000) Anticipatory biasing of visuospatial attention indexed by retinotopically specific alpha-band electroencephalography increases over occipital cortex. J Neurosci 20: RC63(1-6). 